\title{
3D Visual Servo Fuzzy Controller Robust to Displacements of Cameras
}

\author{
Redha Fourar $^{1}$ and Djamel Melaab ${ }^{2}$ \\ Department of Electrical Engineering, Batna University, Algeria \\ ${ }^{1}$ fourarredha@yahoo.fr, ${ }^{2}$ Melaab_djamel@yahoo.fr
}

\begin{abstract}
This paper presents a $3 D$ visual servo system using fuzzy controller for the command of a manipulator arm. The vision system is composed of two cameras; the first is installed above the robot, while the second is located on the lateral side of this one. These two cameras need no calibration, thus we can install them in any position and orientation with just the condition that the joints and the effector of the robot must remain always visible. Servoing is realized without any prior knowledge of the dynamic and geometric models of the robot. Only the positions, in pixels, of the effector and of the different joints, in the two images acquired by the two cameras, are used to realize this servoing. Colors have thus been assigned to the joints and to the effector to determine their positions.
\end{abstract}

Keywords: Artificial Intelligence, Fuzzy Control, Robot Control, Image Processing

\section{Introduction}

Visual control refers to the use of the data recovered from a vision system for controlling the movement of a robot. These data may be provided according to two configurations:

-by a camera directly mounted on the manipulator, it is the movable configuration;

- by cameras mounted in the workspace of the manipulator, in order to monitor its movement, it is the fixe configuration [1].

There exist two basic types of visual servoing systems [2-3]:

-Servoing based on the visual image or Image-based visual servoing (IBVS);

- Servoing based on visual position or Position based visual servoing (PBVS).

The servoing based on the visual image has been proposed by [4].The control law is based on the error between the characteristics of the acquired and desired images and contains none estimation of the position of the target. The servoing based on visual position, estimates the position of the target relative to the camera to send the command to the manipulator robot. It depends on the number of the used cameras and their locations. At least two cameras are needed to achieve a 3D visual servoing [5].

Different types of visual servoing have been proposed [3-5-6]. In [3], a proportional fuzzy distributed control system to guide a redundant manipulator robot, with vision, is proposed. The fuzzy algorithm is used to model the analytical image based on IBVS and the moments of the form are used for the offline learning. [5], has proposed a visual servoing based on images of a manipulator robot with 5 degrees of freedom. The information delivered by the camera is converted to angles by using of the inverse kinematics. These angles are subsequently used to control the manipulator robot. [6], has proposed a visual control algorithm by the use of the 
two servoing IBVS and PBVS. The presented algorithm has a double performance whatsoever in the image space or Cartesian space.

In our work, we have developed a new concept of visual servoing based on fuzzy controller. It controls the different joints of a manipulator arm to lead the effector to a point defined in the 3D space, using images provided by two cameras. The first camera, mounted above the manipulator, will provide to the fuzzy controller the alignment error, in pixels, between the position of the effector and the target. The second camera, mounted on the lateral side of the manipulator, will supply the regulator with the position error, also in pixels, between the effector and target. The servoing based on fuzzy controller provides two different configurations to reach the target, respecting the direction of rotation of each joint while avoiding the collision between the different segments of the manipulator arm. All the images processing necessary to extract, from the two images provided by the two cameras, the informations needed to control the manipulator, is carried out in the MATLAB ${ }^{\circledR}$ environment. The simulation is conducted in the environment of the software SIMULINK ${ }^{\circledR}$ of MATLAB ${ }^{\circledR}$ where we used the virtual reality and the computer vision system toolboxes.

The remainder of this paper is organized as follows. In Sections 2, we present the details of our vision system and the corresponding image processing. In section 3 , we describe the geometric transfer that allows adaptation of the preprocessed images to the fuzzy controllers' inputs. Section 4, focuses on the details of implanted fuzzy controllers. In Sections 5, the mathematical model of robot is discussed and simulation results are presented in Section 6. Finally, Section 7 concludes the paper and suggests future work.

\section{Visual Servo System}

The proposed visual servo system consists of three parts: the first deals with the acquisition and images processing, the second deals with the geometric transfer and finally the last part is devoted to the Fuzzy controller (Figure 1).

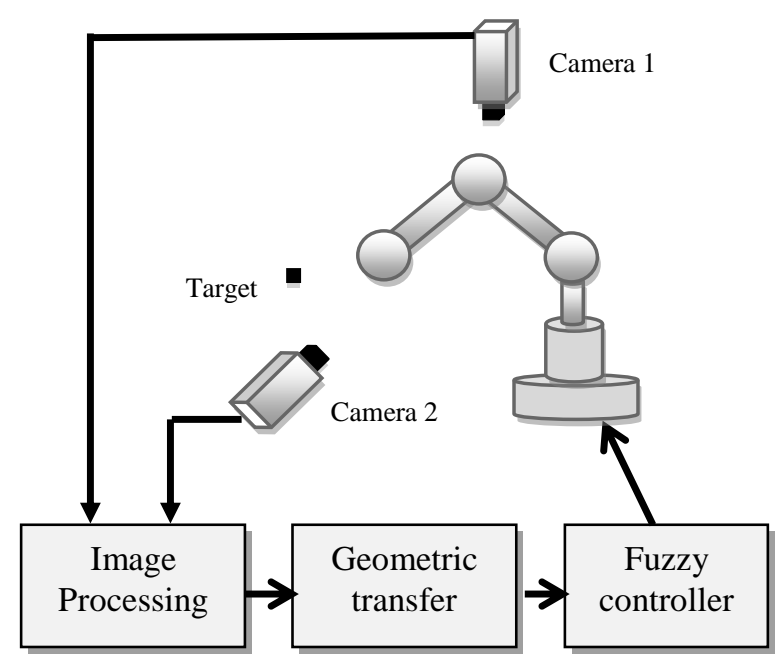

Figure 1. Synoptic of the Visual Servo System

\subsection{Images Acquisition}

Images acquisition was carried out through two cameras installed above (camera1) and on the lateral side (camera 2) of the robot. Each camera delivers a RGB video image of 800x800 
pixels. The VR Sink block, in the Simulink software, allows us to create video images of the virtual reality world as shown in Figure 2.
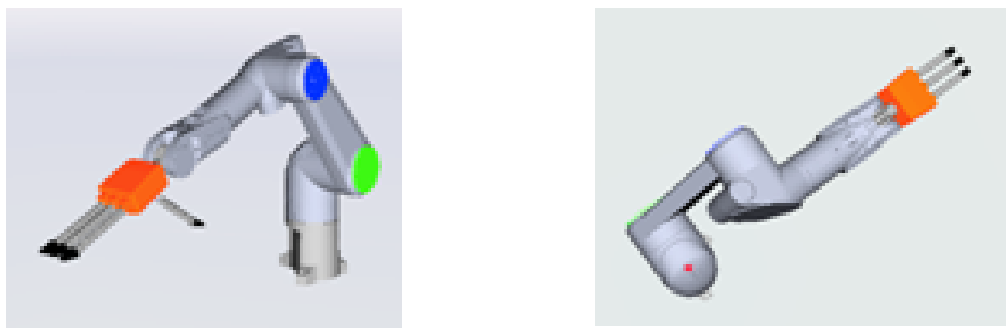

Figure 2. Image of $800 \times 800$ Pixels Provided by the Cameras 1 and 2

With the VR Sink block we can control, the zoom, the orientation, the position and the resolution of the camera. The used robot is the TX60L. This is not at all a limitation of our system. Indeed, the reasoning, applied on this type of robot, remains valid for all other types of robots. Colors were assigned to the joints and the effector to determine their positions in the image space. We attributed the green to the joint 2, the blue to the joint 3 and the red to the joint 1 and the effector. The target is represented by a cyan color.

\subsection{Images Preprocessing}

Detecting the position of the effector and the positions of the different joints consists in detecting the corresponding pixels in the video images acquired according to the steps below:

\subsection{Conversion of Images in the YCbCr Space}

In the $\mathrm{YCbCr}$ space, informations of luminance and chrominance are separated. The parameter Y represents the luminance, that is to say, the information in black and white, while $\mathrm{Cb}$ and $\mathrm{Cr}$ are used to represent the blue and red chrominance, that is to say the information about the color [7]. The expressions of $\mathrm{Y}, \mathrm{Cb}$ and $\mathrm{Cr}$ according to the parameters $\mathrm{R}, \mathrm{G}$ and $\mathrm{B}$ are [7].

$$
\left\{\begin{array}{l}
Y=0.299 * R+0.587 * G+0.114 * B \\
C b=0.1687 * R-0.3313 * G+0.5 * B+128 \\
C r=0.5 * R-0.4187 * G-0.0813 * B+128
\end{array}\right.
$$

These expressions are used to determine the components $\mathrm{Y}, \mathrm{Cb}$ and $\mathrm{Cr}$ from the video images provided by the two cameras.

\subsection{Thresholding and Filtering}

Thresholding the three images, obtained in the previous section, gives three binary images. The application of median filter on these latter eliminates noise of type salt and pepper. Now, binary images corresponding to the $\mathrm{Cb}$ and $\mathrm{Cr}$ components, are used to extract the position of the effector and the positions of all the joints of the manipulator as well as the position of the target. Figure 3, gives an example of the positions of the effector, the target and the joints 2 and 3 , as calculated from the video image provided by camera 2. Position of joint 1 is obtained from images provided by camera 1 . 


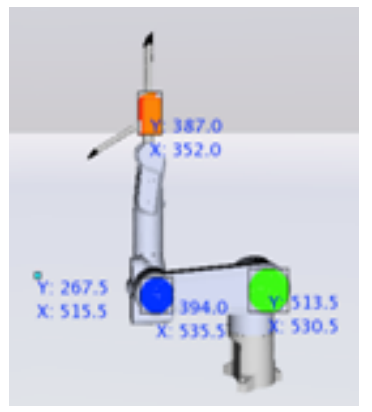

\section{Figure 3. The Effector, the Target and Joints 2 and 3, as Captured by the Camera 2}

\section{Geometric Transfer}

The geometric transfer is a paramount step in the servoing loop. This transfer should allow, in fact, the adaptation of the image processing results, discussed in the previous section, to the inputs of the fuzzy controller. Three geometric transfers are then performed: the first one is performed at the image plane of the camera 1 . The two others are performed at the image plane of the camera 2.

\subsection{Plane of Image of the Camera 1}

Figure 4 shows the position of the effector, the target and the joint 1 as viewed by the camera 1. Let us consider the new frame whose origin is defined by the position of the joint 1 . The ordinate axis $\mathrm{Y}$, is the line joining the joint 1 and the target and directed towards this latter. The abscissa axis $\mathrm{X}$, is perpendicular to axis $\mathrm{Y}$. Let $e$, the error between the $\mathrm{x}$ coordinate of the target and the $\mathrm{x}$ coordinate of the effector. de represents the time derivative of this error. Four cases are considered to ensure that the position of the effector is close to the position of the target and on the same plane as that which contains this target and the origin defined by the joint 1 .

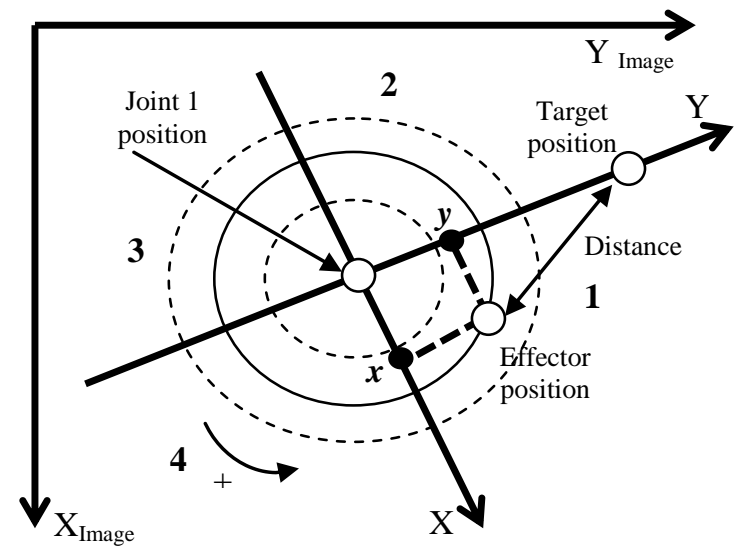

Figure 4. Implementation of the Frame to Execute the Command

Case1 (Figure 5): $\mathrm{x}_{\text {effector }}$ and $\mathrm{y}_{\text {effector }}$ are $>=0$. 


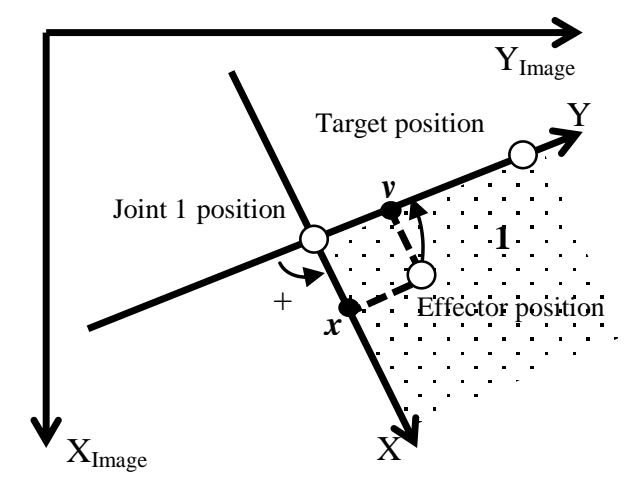

Figure 5. First Case for Control

The sense of rotation of joint 1 must be positive and $d e$ is always negative. Case2 (Figure 6): $x_{\text {effector }}<=0$ and $y_{\text {effector }}>=0$.

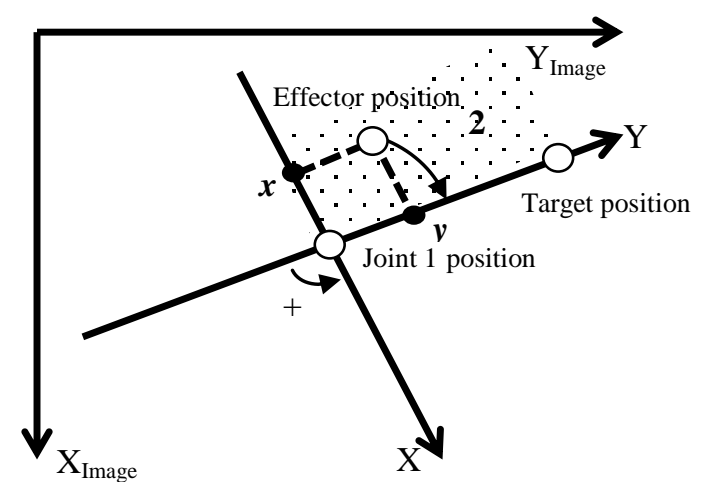

Figure 6. Second Case for Control

Same as case 1 except that the sense of rotation of joint 1 is negative. Case3 (Figure 7): $x_{\text {effector }}$ and $y_{\text {effector }}$ are $<=0$.

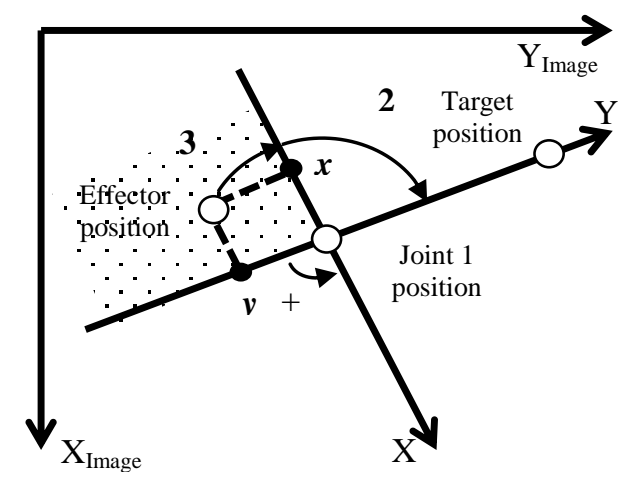

Figure 7. Third Case for Control

In this case it is necessary to move the effector from zone 3 to zone 2.The sense of rotation of joint 1 is negative and $d e$ is always positive.

Case4 (Figure 8): $\mathrm{x}_{\text {effector }}>=0$ and $\mathrm{y}_{\text {effector }}<=0$. 


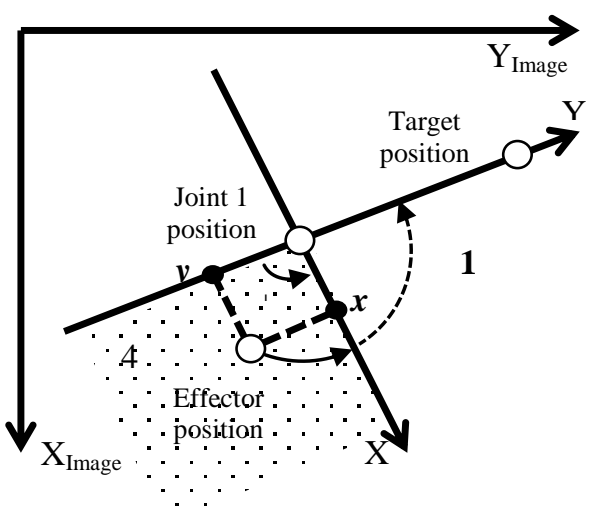

Figure 8. Fourth Case for Control

The effector must be moved from zone 4 to zone 1 . The sense of rotation of joint 1 is positive and $d e$ is always positive.

\subsection{Plane of image of the camera 2}

As previously explained, the servoing performed in the plane of the camera 1 allows to place the joint $\mathrm{q}_{1}$, the effector and the target in the same plane. To complete the task, the servoing performed in the plane of the camera 2 should allow to lead the effector to the target (Figure 9). Let Lv the length in pixels, captured by the camera 2, of the segment between the joint 3 and the effector. L the distance, also in pixels, between the joint 3 and the target as captured by the same camera. Two servoings, running concurrently, are then implemented:

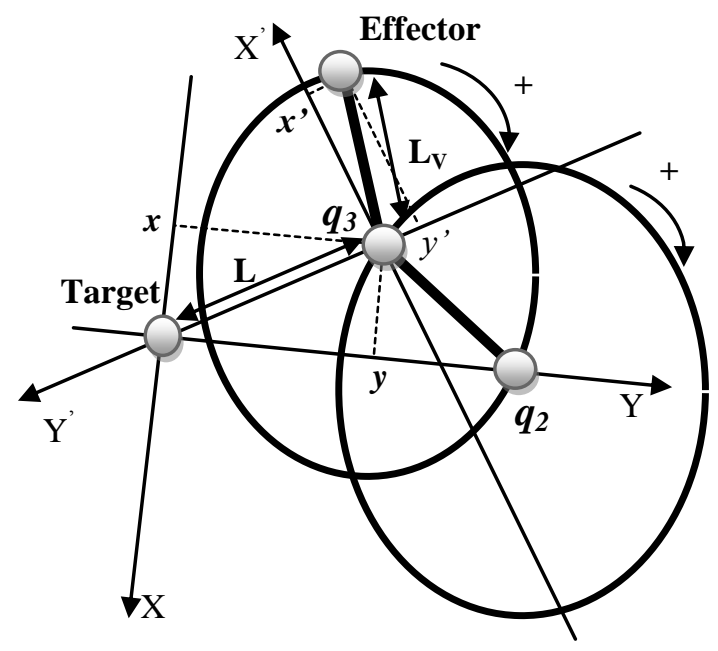

Figure 9. Implementation of Frames to Allow the Command of the joint2

The first acts on joint 2, to position joint 3 at a distance $L_{V}$ with respect to the target. Calculate the difference between the length $\mathrm{L}_{V}$ and the length $\mathrm{L}$ as well as the variation of the absolute value of this difference allows us minimize the error between $\mathrm{L}_{\mathrm{V}}$ and $\mathrm{L}$.

The second acts on joint 3 , to minimize the distance between the target and the effector. As the objective of the control, for the two joints 1 and 3, is to minimize the distance between the target and the effector, then they have the same steps to perform the regulation and the 
geometric transfer. Figure 10 shows the two frames $X^{\prime} Y^{\prime}$ and $X^{\prime \prime} Y$ " used for this purpose. The frame $X^{\prime} Y^{\prime}$ is used to perform the regulation so that the difference, between the $\mathrm{x}$ coordinates of the target and the effector,be equal zero. The frameX" $\mathrm{Y}$ " is used to divide the workspace of the robot into two classes, in order to achieve the geometric transfer that allows us to choose the right direction towards the target considering the mechanical limitations. The effector takes the class 1 or 2 depending on whether its ordinate $\mathrm{Y}^{\prime \prime}$ is $\langle=0$ or $>0$ respectively. The same is for the target.Let $(\mathrm{x}, \mathrm{y})$ the ordinates of the effector, the algorithm to change the frame is given as follows:

$>$ No change of frame if the effector and the target have the same class ;

$>$ If the difference, between the class of the target and the class of the effector, is negative:

- If both $\mathrm{x}$ and $\mathrm{y}$ are positive then the frame $\mathrm{X}^{\prime} \mathrm{Y}^{\prime}$ is rotated by $\mathrm{Pi}$;

- If $\mathrm{X}>0$ and $\mathrm{y}<0$ then the frame $\mathrm{X}^{\prime} \mathrm{Y}^{\prime}$ is rotated by $\mathrm{Pi} / 2$;

$>$ Otherwise no change.

$>$ If the difference, between the class of the target and the class of the effector, is positive:

- If both $x$ and $y$ are negative then the frame $X^{\prime} Y^{\prime}$ is rotated by $-\mathrm{Pi} / 2$;

- If $\mathrm{X}<0$ and $\mathrm{y}>0$ then the frame $\mathrm{X}^{\prime} \mathrm{Y}^{\prime}$ is rotated by $\mathrm{Pi} / 2$;

$>$ Otherwise no change.

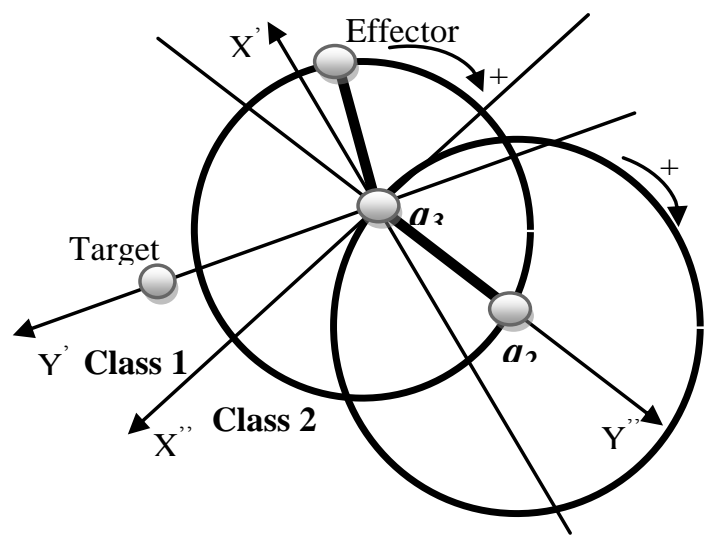

Figure 10. Implementation of Frames to Allow the Command of the joint3

\section{Fuzzy Controllers}

To carry out our task, lead the effector to the target, a set of fuzzy controllers is developed. Our choice fell on this type of controller for its flexibility, robustness and simplicity to implement. Three fuzzy controllers are thus developed. Each controller is assigned to ajoint of our manipulator.

\subsection{Fuzzy Controller for the joint 1}

The structure of the fuzzy controller is shown in Figure 11. It is composed of the following elements:

- Fuzzifier: to pass from physical domain to suitable linguistic values;

- A base of rules: to structure the knowledge that we have on the robot control;

- An inference engine that has the ability to simulate the human decision making. In our case, we have used the most commonly used Max-Min method; 
- Defuzzifier: to transform the fuzzy set to a numeric value. In our case, we have used the most commonly used method of center of gravity.

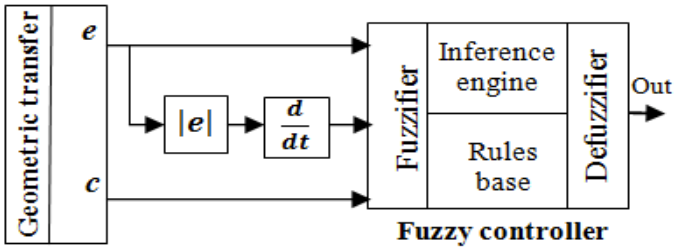

\section{Figure 11. Synoptic of the Fuzzy Controller}

According to the cases treated in paragraph 3.1, the fuzzy controller will receive three inputs: The first one is the error (e) between the position of the target and the position of the effector along the $\mathrm{x}$ axis, the second is the time derivative of this error(de) and a third input (c) which takes the value 1 if $y>=0$ or -1 if $y<0$ where $y$ always designates the ordinate of the effector. The rules base is:

1. if ( $e$ is $\mathrm{N}$ ) and (de is $\mathrm{N}$ ) and ( $c$ is $\mathrm{P}$ ) then (out is $\mathrm{ZE}$ )

2. if ( $e$ is $\mathrm{N})$ and (de is $\mathrm{P})$ and ( $c$ is $\mathrm{P})$ then (out is $\mathrm{P})$

3. if ( $e$ is $\mathrm{P}$ ) and (de is $\mathrm{N}$ ) and ( $c$ is $\mathrm{P})$ then (out is $\mathrm{ZE}$ )

4. if ( $e$ is $\mathrm{P})$ and (de is $\mathrm{P})$ and ( $c$ is $\mathrm{P}$ ) then (out is $\mathrm{N}$ )

5. if ( $e$ is $\mathrm{N}$ ) and (de is $\mathrm{P}$ ) and ( $c$ is $\mathrm{N}$ ) then (out is $\mathrm{ZE}$ )

6. if ( $e$ is $\mathrm{N}$ ) and (de is $\mathrm{N}$ ) and ( $c$ is $\mathrm{N}$ ) then (out is $\mathrm{P}$ )

7. if ( $e$ is $\mathrm{P})$ and (de is $\mathrm{N}$ ) and ( $c$ is $\mathrm{N}$ ) then (out is $\mathrm{N}$ )

8. if ( $e$ is $\mathrm{P})$ and ( $d e$ is $\mathrm{P}$ ) and ( $c$ is $\mathrm{N}$ ) then (out is $\mathrm{ZE})$

The membership functions for the three inputs e, de, and c and for the output "out" are shown in Figure 12.
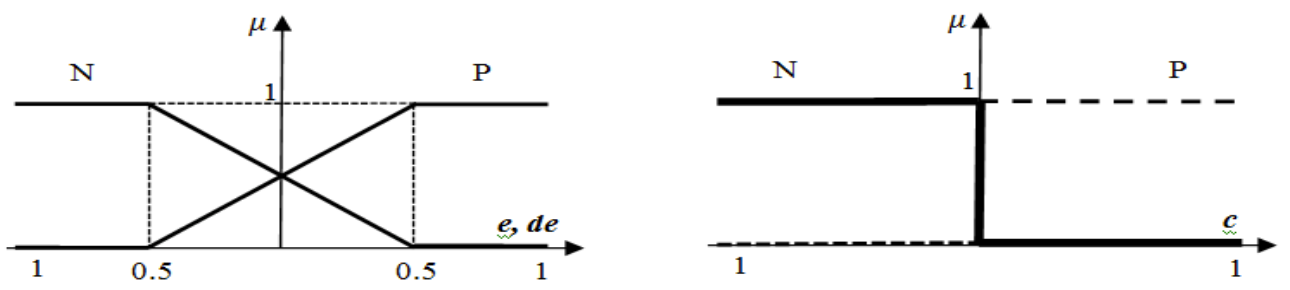

Figure 12. Membership Functions of the Inputs: e, de, c

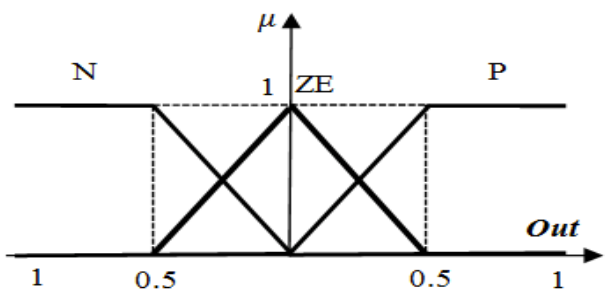

Figure 13. Membership Function of the output "out" 


\subsection{Fuzzy Controller for the joint 2}

The structure and the membership functions are the same as those used for the control of the joint 1 , only rules change. The inputs of the fuzzy controller are: the error e, de and c suh that:

$$
\begin{gathered}
e=L_{V}-L \\
d e=\frac{d\left|L_{V}-L\right|}{d t} \\
c=1, \text { if } x_{j o \text { in } 3} \geq 0 \text {, and } C=-1, \text { if } x_{j o \text { int } 3}<0
\end{gathered}
$$

The rules base is:

1. if ( $e$ is $\mathrm{N}$ ) and (de is $\mathrm{N}$ ) and ( $c$ is $\mathrm{P})$ then (out is $\mathrm{N}$ )

2. if ( $e$ is $\mathrm{N})$ and (de is $\mathrm{P})$ and ( $c$ is $\mathrm{P})$ then (out is $\mathrm{ZE})$

3. if ( $e$ is $\mathrm{P})$ and (de is $\mathrm{N})$ and ( $c$ is $\mathrm{P})$ then (out is $\mathrm{ZE})$

4. if ( $e$ is $\mathrm{P})$ and (de is $\mathrm{P})$ and ( $c$ is $\mathrm{P})$ then (out is $\mathrm{P})$

5. if ( $e$ is $\mathrm{N})$ and (de is $\mathrm{P})$ and ( $c$ is $\mathrm{N}$ ) then (out is $\mathrm{ZE})$

6. if ( $e$ is $\mathrm{N})$ and ( $d e$ is $\mathrm{N}$ ) and ( $c$ is $\mathrm{N}$ ) then (out is $\mathrm{P})$

7. if ( $e$ is $\mathrm{P})$ and (de is $\mathrm{N}$ ) and ( $c$ is $\mathrm{N}$ ) then (out is $\mathrm{ZE}$ )

8. if ( $e$ is $\mathrm{P})$ and (de is $\mathrm{P})$ and $(c$ is $\mathrm{N})$ then (out is $\mathrm{N})$

Two configurations are then envisaged. They are set by the value of $c$. If we want choose the first configuration, we select $e>0$ and $c<0$. If we want choose the second configuration, we select $\mathrm{e}>0$ andc $>0$.

\subsection{Fuzzy Controller for the joint 3}

This controller has the same structure, the same inputs, the same membership functions and the same rules as those used for the control of the joint 1 . We must, however, reverse the direction of rotation in the rules of joint 1 , to get the rules of joint 3 : where out is $\mathrm{N}$ becomes $\mathrm{P}$ and vice versa in the rules of joint 1.

\section{Robot control}

Control of robot manipulator requires efficient determination of the torques applied to each actuator for each point of a predicted trajectory, in real time [8]. The dynamic equation of the robot is given by:

$$
D(q) \ddot{q}+C(q, \dot{q}) \dot{q}+g(q)=\tau
$$

Where : $D(q)$ : Matrix of inertia of the manipulator, $C(q, \dot{q})$ is the vector of Coriolis and centrifugal torques, $g(q)$ is the gravity vector and $\tau$ is the vector of torques. A simple method to control a manipulator robot is the use of the linearization principle, around an operating point. An example of this method is the PD control system with gravity compensation $[9,10]$. An integral term can be added to the PD controller, in order to take into account the forces of gravity that can be considered as a constant disturbance. The result is a PID controller which has the form:

$$
\tau=k_{p}\left(q_{d}-q\right)-k_{v} \dot{q}+k_{I} \int\left(q_{d}-q\right) d t
$$


Where $k_{p}$ and $k_{v}$ are defined positive matrices of gains.If the term $k_{I} \int(-\dot{q}) d t$ is added, we obtain a PI2D controller. The global asymptotic stability (GAS) of the PID controller has been proven by [11], taking into account external disturbances.It has been shown that the PI2D controller could ensure semi-global asymptotic stability (SGAS) in the presence of the gravity and bounded external disturbances [12].

The fuzzy controller, which we developed, directly gives the quantity $\left(q_{d}-q\right)$ from the position of the effector and the positions of the joints provided by the cameras. It ensures an effective control without calculating the rotation angles of the various joints. All the control is reduced to the processing of the pixels of the images provided by the cameras by using a fuzzy controller.

$$
\tau=k_{p} a-k_{v} \dot{q}+k_{I 1} \int(a) d t+k_{I 2} \int(-\dot{q}) d t
$$

Compared with the expression (6) we note that the parameter a replaces the quantity $\left(q_{d}-q\right)$. However, this is only a formal comparison. Indeed, this parameter has nothing to do with quantity $\left(q_{d}-q\right)$ used by classical controllers. It designates the signal provided by the fuzzy controller. The term $k_{I} \int(-\dot{q}) d t$ fixes the controller structure as an PI2D.Thus we call this controller Fuzzy PI2D controller.

\section{Simulation Results}

Figure 14 shows the block diagram of our Fuzzy PI2D controller. This is the coupling between a fuzzy controller and a PI2D control. Figure 15 shows the block diagram of the visual control loop, including the part of the image processing and that of the geometric transfer. The fuzzy control has been tested for different positions of the two cameras and the target. Figure 16 shows an example, of a possible configuration, to reach the target. The other configurations are rejected because of mechanical limitations. Outputs of the fuzzy controller and the rotation angles of the joints 1-3, corresponding to this configuration, are given in Figure 17. Figures 18 and 19 provide respectively the video images acquired and the corresponding control curves, after moving the cameras.

In Figures 16 and 18 the target is not moved. It really occupies the same position in both figures. However, due to the displacement of the cameras in Figure 18, the target is seen by the system as being in a new position. This is reflected in the curves traced by the various joints to reach the target: The curves of Figure 17 are different from those of Figure 19, although the target really occupies the same position in both figures. Indeed, these curves are determined from the lengths of the different segments of the manipulator as seen by the system. As these segments are seen differently, because of the displacement of the cameras, then these curves should be naturally different. However, the end point (target position) is correctly reached in both situations before and after the movement of the cameras. As can be seen in Figures16 and 18, the error between the position of the target and the position of the effector is minimum which shows the efficiency and the robustness of our fuzzy controller relative to the displacement of the cameras. It is also noted that for a direct displacement, while avoiding overruns, we must choose the right position of the cameras. 


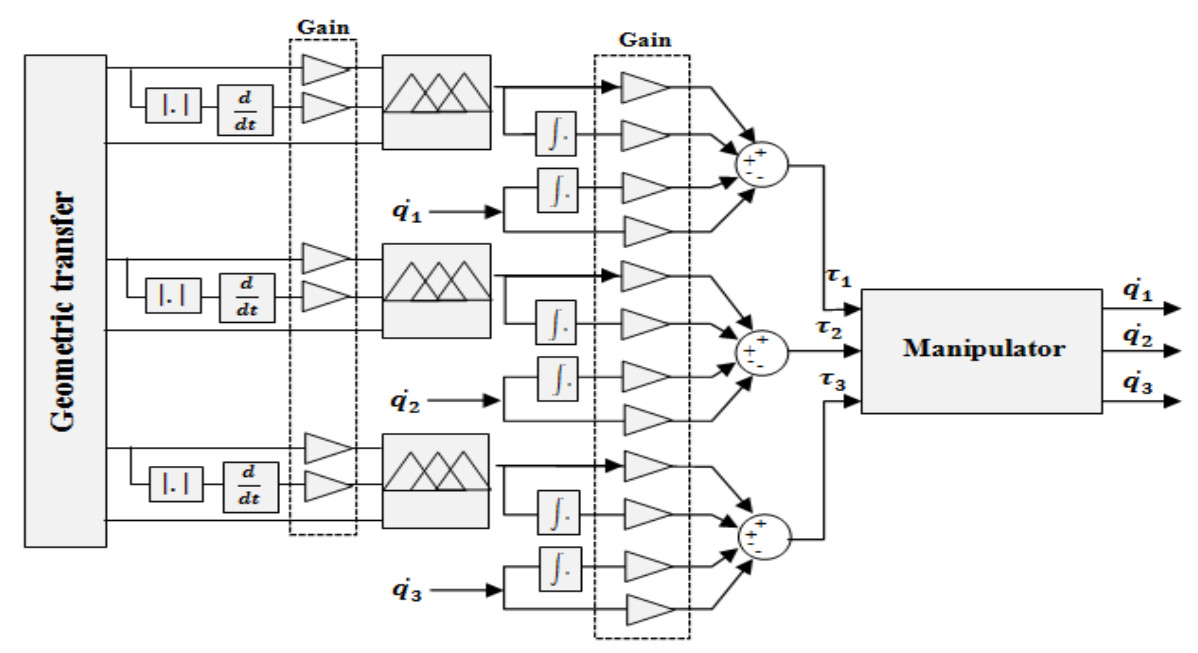

Figure 14. Block Diagram of the Coupling between the Fuzzy Controller and the PI2D Control

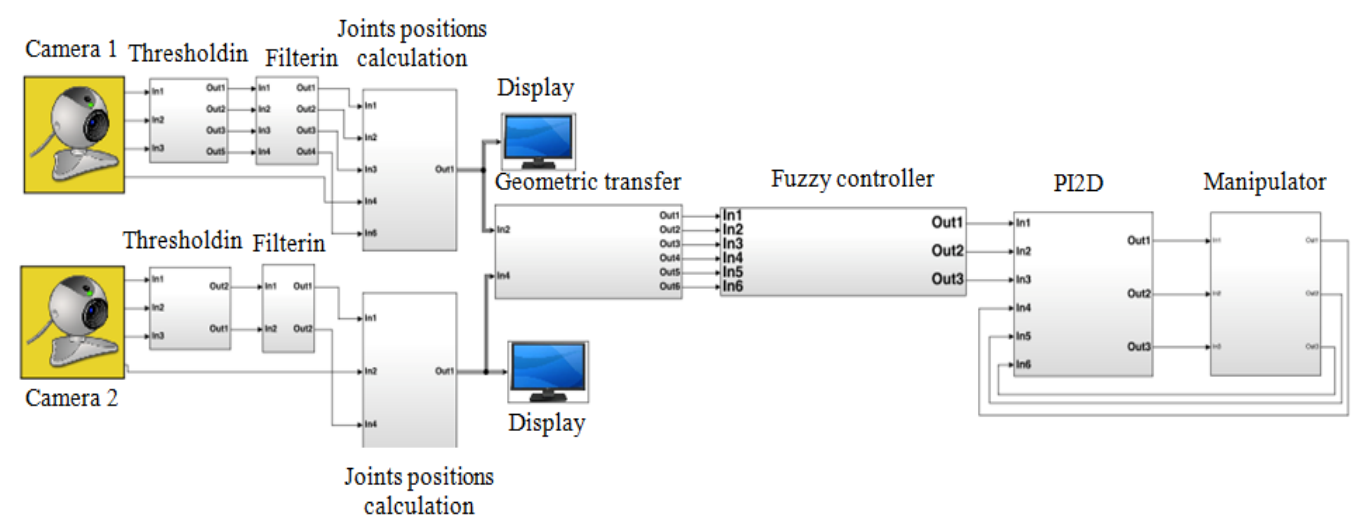

Figure 15. Block Diagram of the Visual Control Loop

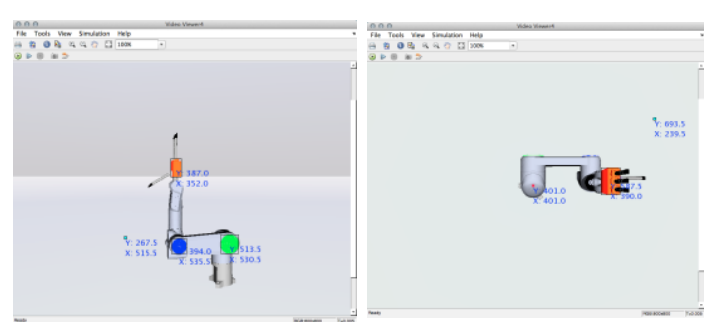

(a)Initial Configuration:Lateral/Top Views

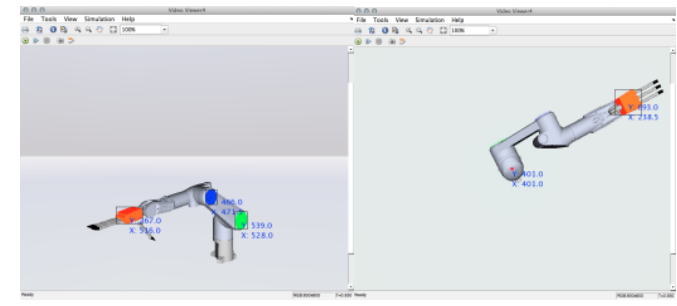

(b) Final Configuration:Lateral/Top Views

Figure 16. Initial and Final Configurations as Viewed by the Cameras 

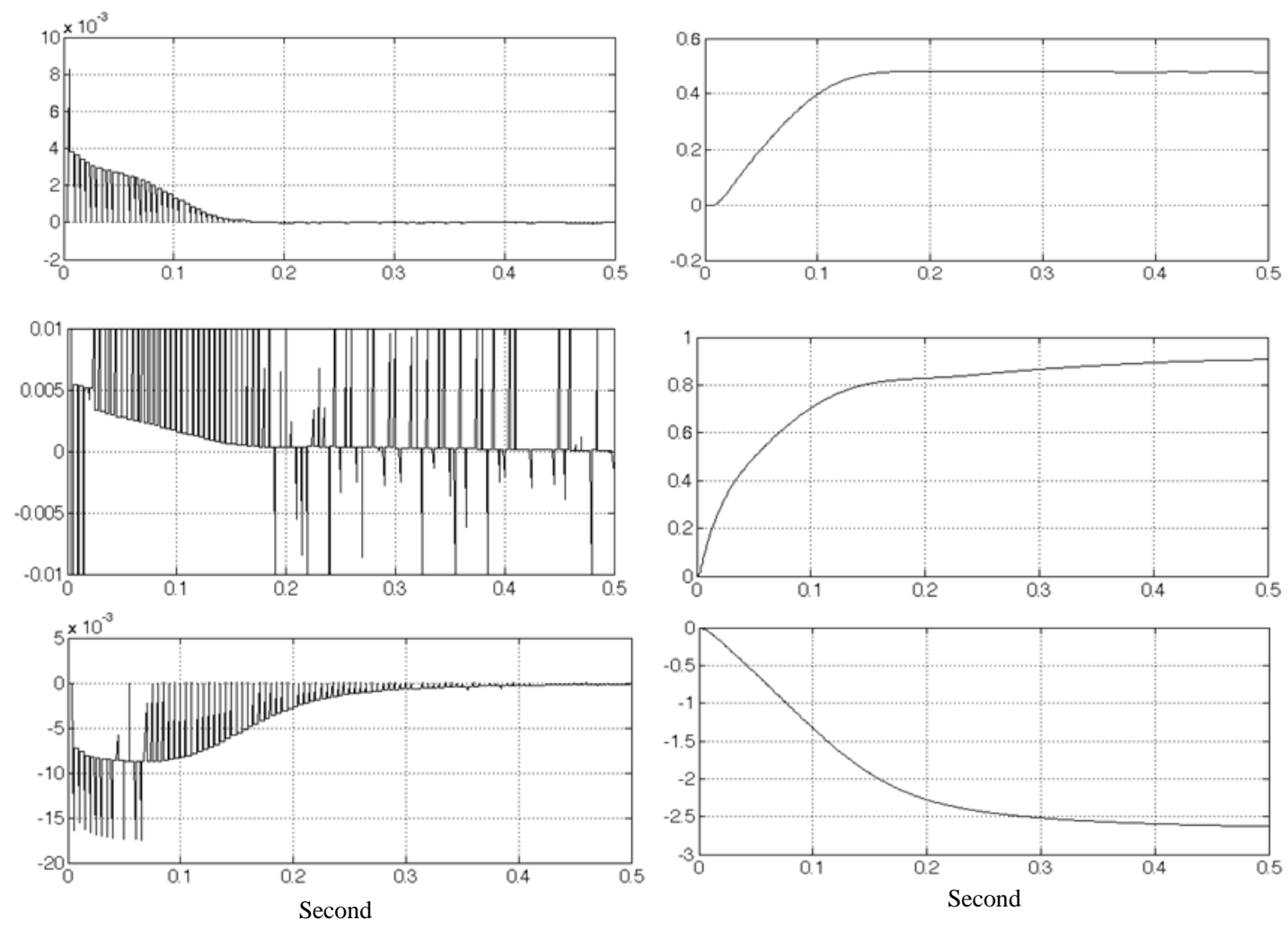

(a) Fuzzy controller outputs for the joints $q 1-q 3$

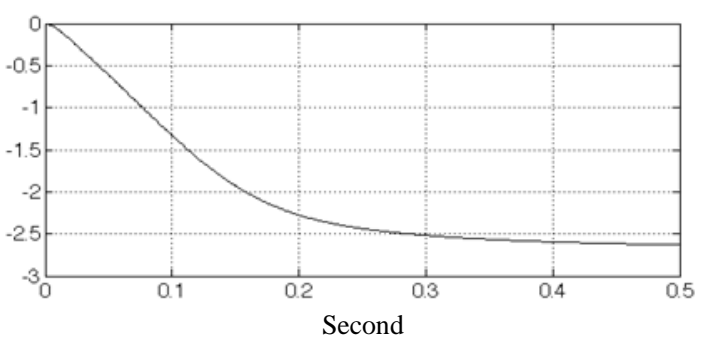

(b) Rotation angles of the joints 1-3 (in radians)

Figure 17. Command Curves

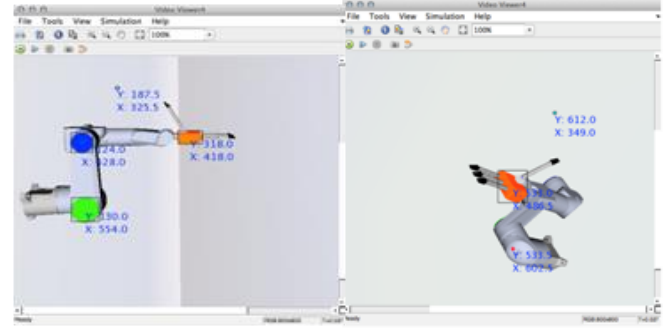

(a) Initial Configuration: Lateral/Top Views

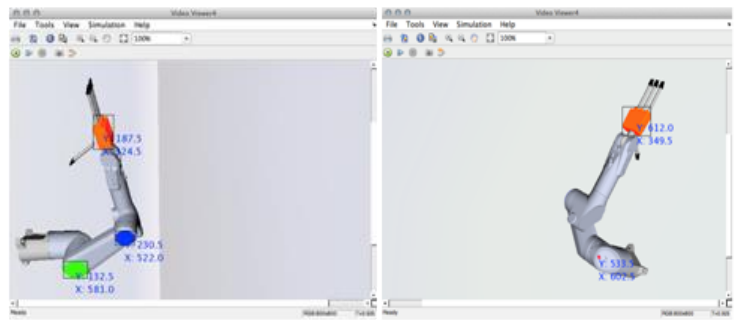

(b) Final Configuration:Lateral/Top Views

\section{Figure 18. Obtained Result, After Moving Cameras}



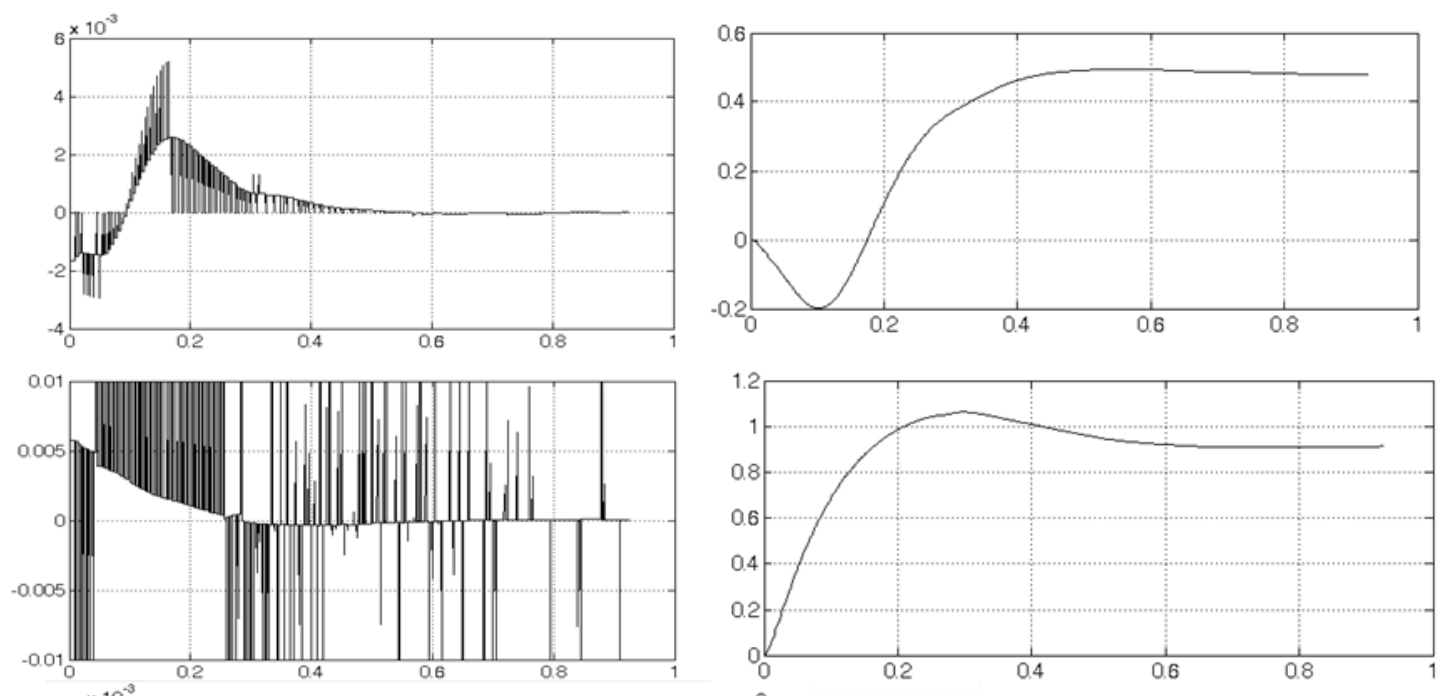

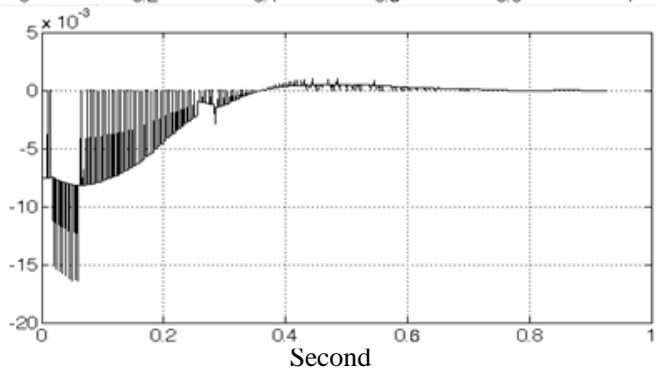

(a) Fuzzy controller outputs for the joints q1-q3

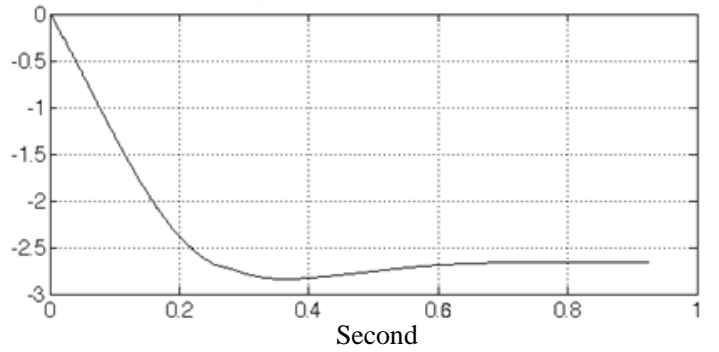

(b) Rotation angles of the joints 1-3 (in radians)

Figure 19. Command Curves Obtained after Moving the Cameras

\section{Conclusion and Future Work}

In this work, we presented a simple and efficient new visual servoing technique for the control of manipulator robot. The command is ensured by a vision system coupled with a dual-controller. The latter consists of a fuzzy controller and a PI2D controller. The resulting system is extremely accurate to lead the manipulator to a target. This precision is even in the order of the pixel. No prior knowledge of the dynamic and geometric models of the robot is required, which attribute to the system remarkable simplicity. In this work, we have developed only position servoing, future work will focus on adding an visual orientation servoing to allow grasping the objects by the manipulator.

\section{References}

[1] F. Chaumette and S. Hutchinson, Springer Handbook of Robotics. Springer-Verlag Berlin Heidelberg, 2008, ch. 24. Visual Servoing and Visual Tracking.

[2] P. I. Corke, "Visual control of robot manipulators", Visual Servoing, World Scientific, (1994), pp. 1-31.

[3] I. Siradjuddin, L. Behera, T. M. McGinnity and S. Coleman, "Image Based Visual Servoing of a 7 DOF Robot Manipulator Using a Distributed Fuzzy Proportional Controller”, Fuzzy Systems (FUZZ), IEEE International Conference, (2010) July 18-23, pp. 1-8.

[4] A. C. Sanderson and L. E. Weiss, "Adaptive visual servo control of robots", A. Pugh, editor, Robot Vision, pp. 107-116. IFS, (1983). 
[5] S. Y. Park, Y. J. Kim and J. J. Lee, "Controlling Robot Arm Manipulator Using Image-Based Visual Servoing without Pre-Depth Information”, 37th Annual Conference on IEEE Industrial Electronics Society, (2011) pp. 3276-3280.

[6] J. H. Aabdul Hafez, E. Cervera and C. V. Jawahar, "Hybrid Visual Servoing by Boosting IBVS and PBVS", Information and Communication Technologies: From Theory to Applications", 3rd International Conference, (2008) April 7-11, pp. 1-6.

[7] M. Xie, J. Wu, L. Zhang and C. Li, "A Novel Boiler Flame Image Segmentation and Tracking Algorithm Based on YCbCr Color Space”, International Conference on Information and Automation, ICIA '09”, (2009) pp. 138-143.

[8] A. Katbab, "Nonlinear Adaptive Control of Robotic Manipulators - Hyperstability Approach", Robotics and Autonomous Systems. vol. 4, (1988), pp. 265-273.

[9] R. Kelly, "PD control with desired gravity compensation of robot manipulators", Int. J. Robot. Res., vol. 16, no. 5, (1997), pp. 660-672.

[10] M. Takegaki and S. Arimoto, "A new feedback method for dynamic control of manipulators", Trans. ASME J. Dyn. Syst. Meas. Contr., vol. 102, (1981), pp. 119-125.

[11] L. C. Fu, "Robust adaptive decentralized control of robot manipulators", IEEE Trans. Autom. Contr., vol. 37, no. 1, (1992), pp. 106-110.

[12] R. Ortega, A. Loria and R. Kelly, "A semi-globally stable output feedback PI2D regulator for robot manipulators”, IEEE Trans. Autom. Contr., vol. 40, no. 8, (1995), pp. 1432-1436.

\section{Authors}

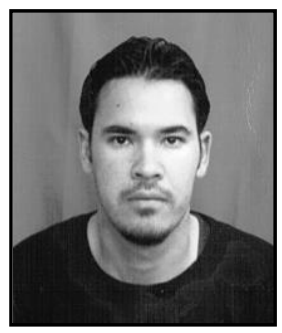

Redha Fourar was born in Batna, Algeria in 1983. He received his Engineering Master degree and magister in Robotics from University of Batna, Algeria. Currently, he is a PhD student. His interests include digital image processing, robotics and artificial intelligence, signal processing.

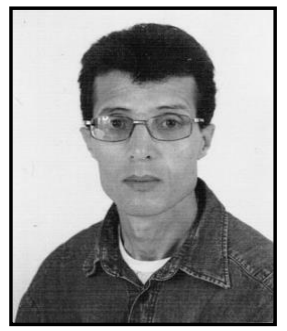

Djamel Melaab received his Ph.D. in Automatic from the University of Bordeaux, France in 1991. He is currently lecturer at the University of Batna, Algeria. He works in the field of artificial intelligence, and Robotics. His current research interests mainly include the application of artificial intelligence techniques in the field of biometric recognition, Biomedical Engineering and Robotics. 